\title{
Variable Selection in Structural Equation Models with Regularized MIMIC Models
}

\author{
Ross Jacobucci ${ }^{\mathrm{a}}$, Andreas M. Brandmaier ${ }^{\mathrm{b}, \mathrm{c}}$, Rogier A. Kievit ${ }^{\mathrm{c}, \mathrm{d}}$ \\ ${ }^{\text {a }}$ University of Notre Dame, Indiana, USA \\ ${ }^{\mathrm{b}}$ Max Planck Institute for Human Development, Berlin, Germany \\ ${ }^{\mathrm{c}}$ Max Planck UCL Centre for Computational Psychiatry and Ageing Research Berlin, Germany / \\ London, UK \\ ${ }^{\mathrm{d}}$ Medical Research Council Cognition and Brain Sciences Unit, University of Cambridge, UK
}

Author Note

RAK is supported by the Sir Henry Wellcome Trust (grant number 107392/Z/15/Z) and MRC Programme Grant SUAG/014/RG91365. This project has also received funding from the European Union's Horizon 2020 research and innovation programme (grant agreement number 732592). 


\begin{abstract}
Methodological innovations have allowed researchers to consider increasingly sophisticated statistical models that are better in line with the complexities of real world behavioural data. However, despite these powerful new analytic approaches, sample sizes may not always be sufficiently large to deal with the increase in model complexity. This poses a difficult modeling scenario that entails large models with a comparably limited number of observations given the number of parameters (also known as the "small n, large p" problem). We here describe a particular strategy to overcoming this challenge, called regularization. Regularization, a method to penalize model complexity during estimation, has proven a viable option for estimating parameters in this small $\mathrm{n}$, large $\mathrm{p}$ settings, but has so far mostly been used in linear regression models. Here we show how to integrate regularization within structural equation models, a popular analytic approach in psychology. We first describe the rationale behind regularization in regression contexts, and how it can be extended to regularized structural equation modeling (Jacobucci, Grimm, \& McArdle, 2016). Our approach is evaluated through the use of a simulation study, showing that regularized SEM outperforms traditional SEM estimation methods in situations with a large number of predictors, or when sample size is small. We illustrate the power of this approach in a $\mathrm{N}=627$ example from the CAM-CAN study, modeling the neural determinants of visual short term memory. We illustrate the performance of the method and discuss practical aspects of modeling empirical data, and provide a step-by-step online tutorial.

Keywords: regularization, structural equation models, MIMIC, LASSO, variable selection
\end{abstract}




\section{Introduction}

The empirical sciences have seen a rapid increase in data collection, both in the number of studies conducted and in the richness of data within each study. With large numbers of variables available, researchers often seek to explore which variables explain observed variability beyond what their hypothesis-driven models attempted to confirm. Having a large number of variables, whose influence on the variables or constructs of interest is unclear, researchers face the problem of selecting the variables that are most informative about the outcome of interest. Typical questions asked are: "What is the importance of my variables for predicting the outcome of interest?" and, ultimately, "What subset of variables is most predictive about (or most relevant for) my outcome?"

How to perform variable selection is pervasive challenge in applied statistics. The field of statistical learning (also known as 'machine learning' or 'data mining') has dedicated a large amount of attention to the topic of how predictors can be optimally selected when there is little or no prior knowledge. Statistical approaches to variable selection range from the notorious stepwise variable selection procedures (cf. Thompson, 1995) to more complex and comprehensive approaches such as support vector machines or random forests. One particularly fruitful approach is that of regularized regression, a method that solves the variable selection problem by adding a penalty term that penalizes solutions, effectively producing sparse solutions in which only few predictors are allowed to be "active." Regularization approaches vary in their precise specifications and include method such as Ridge (Hoerl \& Kennard, 1970), and Lasso regression (Tibshirani, 1996), as well as the Elastic Net (Zou \& Hastie, 2005) have gained high practical relevance and utility. 
Despite their strengths, these regularization approaches are generally developed in a context of models that only include observed indicators, which do not allow for modeling measurement error. However, incorporation of measurement error is central to many approaches in psychology. The most dominant approach to doing so in psychology and adjacent fields is the use of Structural Equation Modeling. SEM offers a general framework in which hypotheses can be formulated at the construct (latent) level with explicit measurement models linking the observed variables to latent constructs. Latent variable models allow account for measurement error, the assessment of reliability and validity, and often have greater validity, generalizability and statistical power than methods based on observed variables (e.g., Brandmaier, Wenger, Raz, \& Lindenberger, submitted; Little, Lindenberger, \& Nesselroade 1999). Here we describe a novel approach called regularized SEM, which incorporates the strengths of regularization into the SEM framework, allowing researchers to estimate sparse model solutions and implicitly solve large-scale variable selection in structural equation models (SEM) by introducing a penalized likelihood function. Using simulations and data from the CAM-CAN study, in which we examine the neural determinants of visual short term memory, we illustrate the performance of the method and discuss practical aspects of using the method for modeling empirical data. First we outline the general principles of regularization, how to extend these principles to SEM, and show how regularization is a viable and underused tool for settings with large numbers of predictors or relatively small sample sizes.

\section{Regularization Overview}

Regression. To set the stage for discussing the use of regularization (e.g. shrinkage or penalized estimation) in structural equation models, we give a brief overview in the context of regression. For more detail, interested readers may consult McNeish (2015) or Helwig (2017). 
We use ordinary least squares (OLS) estimation as a basis. Given $N$ continuous observations of $p$ predictors in matrix $X$ and associated continuous outcomes $Y$, we can estimate the regression coefficients by minimizing the residual sum of squares

$$
R S S=\sum_{i=1}^{N}\left(Y_{i}-\beta_{0}-\sum_{j=1}^{p} \beta_{j} X_{i j}\right)^{2}
$$

For coefficients, we estimate an intercept $\beta_{0}$ along with $\beta_{j}$ coefficients for each of the $p$ predictors. However, there may be instances when we prefer a simpler model, namely a model that includes fewer predictors of the outcome. To perform variable selection we can use the Least Absolute Shrinkage and Selection Operator (Lasso; Tibshirani, 1996). Lasso regularization builds upon equation 1 above, building in a penalty for each parameter (with larger parameter values incurring a larger penalty):

$$
\text { Lasso }=\underbrace{R S S}_{\text {OLS }}+\underbrace{\lambda \sum_{j=1}^{p}\left|\beta_{j}\right|}_{\text {Lasso }} .
$$

The Lasso penalty includes the traditional RSS as in equation 1, but introduces two new components. First and foremost, it introduces a new penalty term that reflects the sum of all beta coefficients (righthand term in equation 2). In this manner, much like a traditional regression attempts to minimize the squared residuals, the Lasso penalty also tries to drive parameters to zero, thus implicitly performing variable selection. Second, as can be seen in equation 2 , the sum of the absolute value of each $\beta_{j}$ coefficient is multiplied by a hyper-parameter, $\lambda$. This term $\lambda$ quantifies the influence of the Lasso penalty on the overall model fit and thus weights the importance of the least-squares fit versus the importance of the lasso penalty - As $\lambda$ increases, a stronger penalty is incurred for each parameter, which results in greater shrinkage of the coefficient sizes. $\lambda$ is called a hyper-parameter because it cannot be estimated jointly with the $\beta_{j}$ coefficients. As there is no generally optimal value for $\lambda$, is common to test a range of $\lambda$ values, combined with cross-validation, to examine what the most appropriate degree of regularization is 
for a given dataset. An additional type of regularization is Ridge regularization (Hoerl \& Kennard, 1970), where in contrast to the Lasso, the Ridge sums the squared coefficients. Where the Lasso penalty will push the betas all the way to 0 (as any non-zero beta will contribute to the penalty term), the Ridge penalty will instead shrink betas, but not necessarily all the way to 0 (as the squaring operation means that small betas incur negligible penalties). One benefit of Ridge regularization is that it better handles multicollinearity among predictors. In an effort to combine both the variable selection aspects of the Lasso along with the ability to handle collinearity from Ridge regularization, Zou and Hastie (2005) proposed the Elastic Net. Through the use of a mixing parameter, $\alpha$, the Elastic Net combines both Ridge and Lasso regularization

$$
\text { Elastic Net }=R S S+(1-\alpha) \underbrace{\lambda \sum_{j=1}^{p} \beta_{j}^{2}}_{\text {Ridge }}+\alpha \underbrace{\lambda \sum_{j=1}^{p}\left|\beta_{j}\right|}_{\text {Lasso }} .
$$

In the same way that it is common to test different values of $\lambda$, combined with cross-validation to choose a final model, the same can be done for $\alpha$. Generally, this means testing values ranging from zero (equivalent to the Ridge penalty) to 1 (equivalent to the Lasso penalty).

Instead of the traditional use of a test statistic (and associated p-value) to determine the significance of a parameter, we would instead test a sequence of penalties, use model comparison to choose a best fitting model, and examine whether the regression parameter estimates in this best model are non-zero. Non-zero coefficients can be thought of as important (e.g. see Laurin, Boomsma, \& Lubke, 2016). This stands in stark contrast to the use of p-values, as using regularization to label parameters as important does not rely on any asymptotic foundations (it does not make statements with regards to a population). In particular, since the regularized estimates move away from the point of maximum likelihood, asymptotic distributions of parameter estimates do not hold anymore. Commonly paired with cross-validation, regularization 
attempts to identify which parameters are likely to be non-zero not only in the current sample, but also in a holdout sample.

One implicit conceptual assumption of regularization methods that set parameters to zero, such as the Lasso, is that of sparsity (e.g. Hastie, Tibshirani, \& Wainwright, 2015) - In other words, it reflects the hypothesis that the true underlying model has few non-zero parameters. However, in psychological research, this is unlikely to be true. Instead, most variables in a dataset probably have small correlations among themselves (e.g. the "crud" factor; Meehl, 1990). As a result, the use of regularization in psychological research will impart some degree of bias into the results - As with all procedures, there is no such thing as a free lunch (Wolpert \& Macread, 1997 ). Although this may first seem to be an undesirable side effect, we argue that there are common situations where the benefits of reduced variance outweigh the drawbacks of non-zero degrees of bias. First, even though there may be a confluence of small effects in our dataset, we may not value the inclusion of every non-zero parameter into our model, as it complicates estimation and renders interpretation difficult. In this case we care more about what could be termed as functional sparsity, where we specifically aim to develop a parsimonious model that facilitates interpretation and generalization of the most important parameters. Second, one of the main motivations for the development of regularization methods is for datasets that have a larger number of variables than total observations. In this case, OLS regression cannot be used. Although settings where the number of parameters exceeds $\mathrm{N}$ may still be uncommon, the benefits generalize to settings where the ratio of predictors to observations, which can be construed as sample size challenge (e.g. Bakker, Van Dijk, \& Wicherts, 2012). To achieve adequate power to detect a given parameter, a suitably large sample size (depending on the magnitude of the effect) is required. When multiple effects are considered, either separately or in 
the context of a multivariate model, the sample size to detect multiple effects can rapidly increase, reducing power. If collecting additional data is not possible for practical or principled purposes, one strategy for testing complex models in the presence of a small sample is to reduce the dimensionality of the model. Most commonly this means using some method such as stepwise regression to reduce the number of coefficients in a regression model, which can be highly problematic (e.g. Harrell, 2015).

Structural Equation Modeling. Going beyond situations where researchers only have a single continuous outcome, regularization has been extended in a number of directions. This includes generalized linear models (e.g. Park \& Hastie, 2007), network based models (e.g. Epskamp, Rhemtulla, \& Borsboom, 2016), and factor analysis (e.g. Hirose \& Yamamoto, 2015), to name just a few. Specific to our purposes is detailing what we refer to as regularized structural equation modeling (RegSEM; Jacobucci, Grimm, \& McArdle, 2016).

RegSEM directly builds in different types of regularization into the estimation of structural equation models, by expanding the traditional Maximum Likelihood estimation (MLE) to include a penalty term, as follows:

$$
F_{\text {regsem }}=\underbrace{\log (|\Sigma|)+\operatorname{tr}\left(C * \Sigma^{-1}\right)-\log (|C|)-p}_{M L E}+\underbrace{\lambda P(\cdot)}_{\text {penalty }}
$$

This adds a penalty term, $\lambda P(\cdot)$ to the traditional MLE fit function. Just as in regularized regression, $\lambda$ is the penalty, while $P(\cdot)$ is a general function for summing parameters. In the case of the Lasso, $P(\cdot)$ sums the absolute values of the specific parameter estimates. The same goal is accomplished for Ridge penalties, the Elastic Net, as well as other extensions (See Jacobucci, 2017). The other component of $P(\cdot)$ is selecting which parameters estimates should be included (i.e. which parameters are penalized). Because this form of regularization takes place in the estimation of structural equation models, regularization can be selectively applied to subset(s) of 
parameters, including factor loadings (e.g. subset selection in a questionnaire to create a short form), variances or covariances (e.g. test whether the addition of residual covariances is necessary) or, our specific interest, regression paths ${ }^{3}$.

RegSEM combines both confirmatory aspects of structural equation modeling with an exploratory search for important predictors. The confirmatory and exploratory aspects can take place in either the measurement or structural parts of a structural equation model. In many situations, researchers may have some a priori idea of how some variables relate to each other. To be more concrete, this may take the form of a confirmatory factor analysis (CFA) model. For instance, imagine a model with four indicators of a single latent variable such as fluid intelligence. This confirmatory formulation may be the result of previous research support for a single latent dimension underlying the covariance between all of the indicators. In contrast, we may have less certainty about which covariates in our dataset may be important predictors of the fluid intelligence latent factor, either because we lack strong a priori expectations, or because a large number of potential covariates is available (e.g. genetic markers, brain variables). As an example, Figure 1 displays the addition of three predictors (say, volumetric measures of different brain regions, cf. Kievit et al., 2014) to the initial CFA model resulting in a Multiple Indicator, Multiple Causes Model (MIMIC; Joreskog \& Goldberger, 1975). Once the model is run, researchers commonly rely on traditional techniques such as the Wald test (and associated test statistics) to determine which predictors have non-zero population values. This kind of model is commonly used to simultaneously estimate the joint influence of a set of presumed causal influences on one or more latent variables. However, given the constraints of traditional SEM approaches, the predictors are usually selected a priori based on theoretical or empirical considerations (cf. Kievit et al., 2014). Now imagine an alternative scenario, instead of only 
incorporating a small set of predictors in a MIMIC model, researchers may have a much larger number of predictors they may wish to test (such as grey matter volume across all regions in an atlas). None of these additional relationships may be based on previous hypotheses. Instead, an exploratory search would be conducted. Here is where traditional tools are no longer as suitable tools, as the model may not converge, or estimates may be imprecise. This can be attributed to problems in using maximum likelihood estimation (MLE) with large numbers of variables when the sample size is limited (e.g. see Hastie, Tibshirani, \& Wainwright, 2015). Although previous research has examined the influence of large models on test statistics (Yuan, Yang, \& Jiang, 2017), less attention has

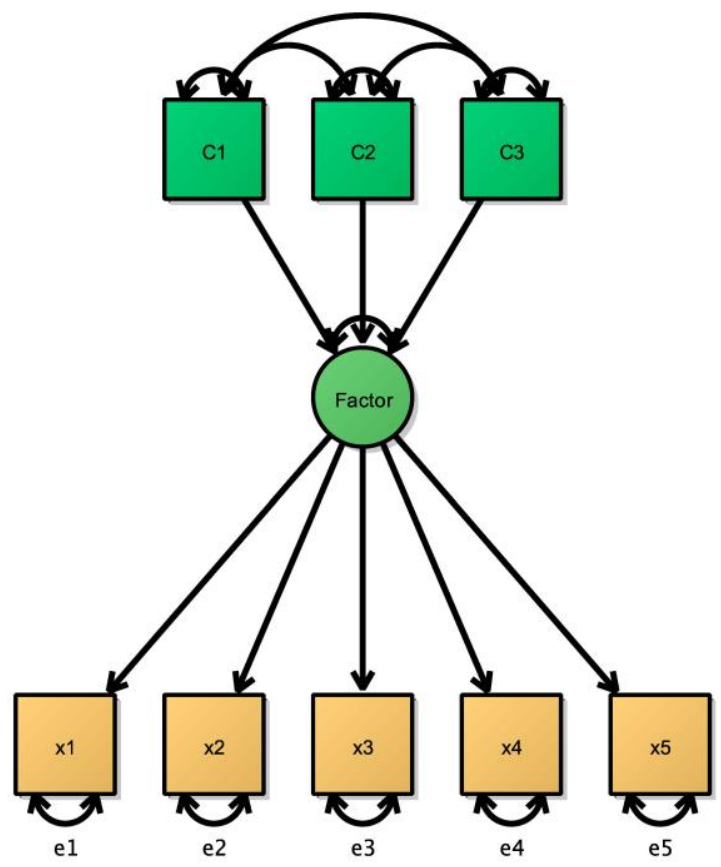
been paid to strategies that produce more accurate parameter estimates. To address Figure 1: Simple MIMIC model (multiple indicators Y, multiple causes $\mathrm{C}$ ).

this challenge, we propose and evaluate the use of regularization to reduce the dimensionality of the model to improve the parameter estimate accuracy.

Combining what we have detailed with regularized regression, the rationale for using regularization, and regularization in structural equation models, we can now revisit our example in Figure 1. In going from the CFA model to the MIMIC model, we transition from a confirmatory latent variable model, based on previous research, to the inclusion of predictors that may not have a strong a priori basis. Moreover, in many applied fields such as genetics, 
cognitive neuroscience, epidemiology and similar fields, the ratio of predictors may be large compared to available sample size number. Indeed, one could argue that the absence of regularization methods may help explain why fields such as cognitive neuroscience to rely on mass univariate approaches (i.e., a relationship between an outcome and neural data is tested thousands of times, separately for each brain region). However, as multivariate approaches generally paint a richer, more realistic picture of the true data structure, as well as allowing the researcher to investigate which effects are redundant across brain regions, and which may be partially independent complementary effects instead. To examine the possible benefits of regularization in the SEM context we conducted two studies. In Study 1 we examine the effectiveness of both MLE and regularization in the context of complex structural equation models. In Study 2 we apply regularized SEM to a large existing dataset.

\section{Study 1: Simulation}

\section{Methods}

To evaluate the effectiveness of the RegSEM Lasso, we designed simulation conditions that researchers may commonly face when evaluating a large number of predictors (e.g., a property such as cortical thickness measured across many brain regions). We vary our simulations across two dimensions: sample size and predictor collinearity. The template model with each simulated parameter is depicted in Figure 2 below. In this, there are six indicators ( $Y 1$ Y6) of the latent variable, $f$. These factor loadings differ in their simulated population values (see Figure 2). As predictors of $f$, there are 70 uninformative ("noise") variables $\left(C_{n 1}-C_{n 70}\right)$, with simulated population coefficients of zero. Additionally, there are three sets of 10 predictors each of differing effect sizes: small $\left(0.20, C_{s 1} C_{s 10}\right)$, medium $\left(0.50 ; C_{m 1}-C_{m 10}\right)$, and large $\left(0.80 ; C_{l 1^{-}}\right.$ $C_{l 10}$ ). Taken together, this makes a dataset of 100 potential predictors of $f$, each treated as fixed 
effects. In fitting this model, the latent variable variance was fixed to one for identification purposes, allowing each factor loading to be freely estimated (we do not estimate a mean structure).

After creating simulated data according to the model in Figure 2, we then tested a model that included 112 free parameters, including one hundred latent regression coefficients, 6 factor loadings, and 6 residual variances. Although rules of thumb are inherently limited, common guidelines would suggest a ratio of 10:1 for sample size, suggesting a minimum $\mathrm{N}$ of 1,200 (e.g. Kline, 2015) to obtain stable estimates. Given that many researchers may wish to test models of this size, but may not have the requisite sample size, we aimed to test a variety of sample sizes to

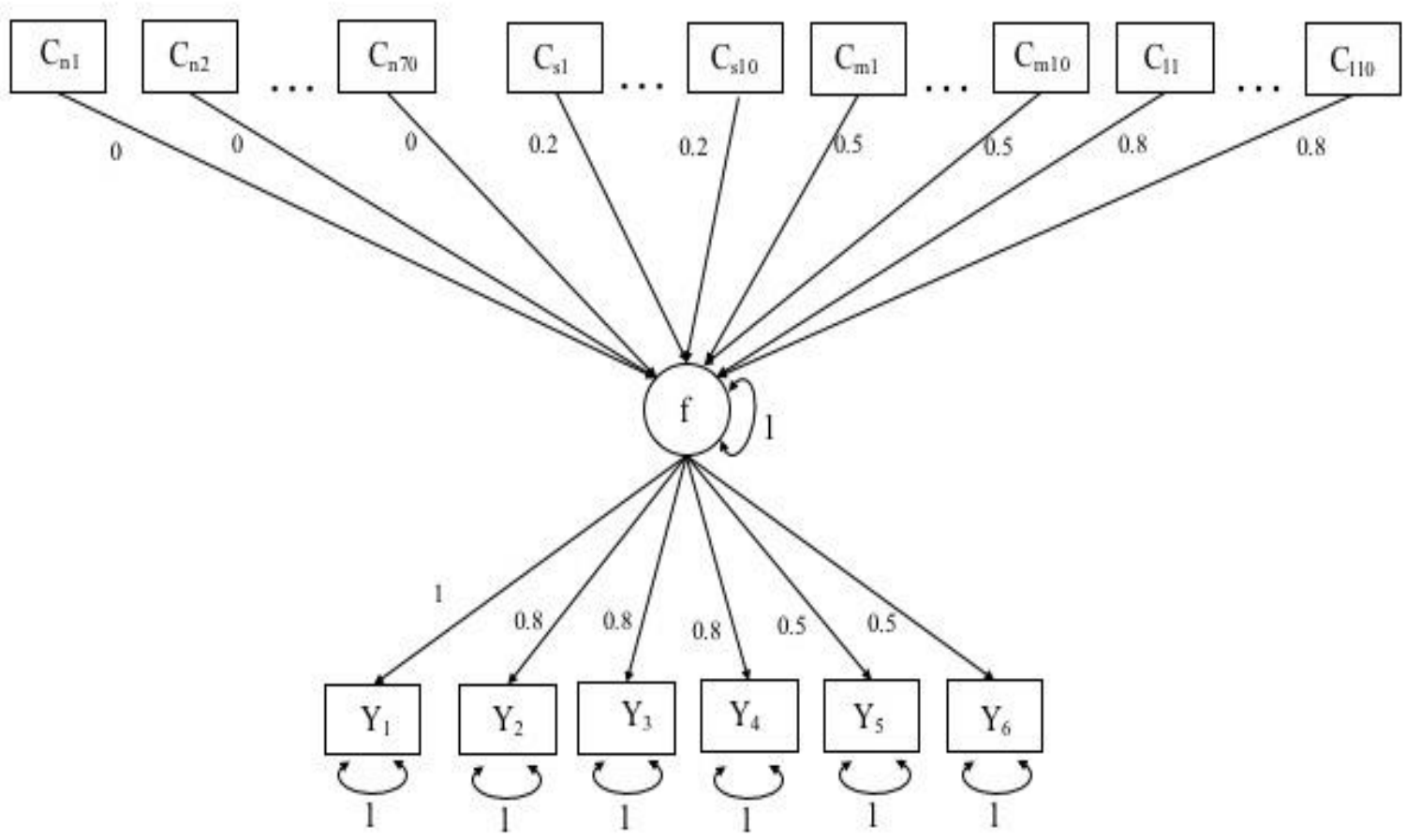

Figure 2: Template simulation model. The model is a MIMIC model including a single latent factor " $\mathrm{f}$ ", six indicators ( $\mathrm{Y} 1$ to $\mathrm{Y} 6$ ) with factor loadings between .5 and 1 and unique error variances, as well as hundred potential predictors. The predictors are either uninformative ( $\mathrm{Cn} 1$ to $\mathrm{Cn70}$ ), have a small effect (Cs1 to examine when the performance of MLE degrades. and when the use of regularization is beneficial. As a result, we tested sample $\operatorname{sizes}^{1}$ of 150, 250, 350, 500, 800, and 2000. 
Finally, in most psychological studies that examine the influence of a variety of predictors it is common that these predictors have correlations amongst themselves. This complicates the interpretation of the results - For instance, it becomes challenging to determine the relative contribution of individual predictors (Grömping, 2009). Moreover, high degrees of collinearity can result in problematic estimation. As a result, we also included predictor collinearity as a simulation condition. To investigate the effect of predictor collinearity, we simulated data that included correlations of $0, .20,0.50,0.80$, and 0.95 among all predictors. With increasing correlation, we expected increasing amounts of bias in both MLE and regularized estimation. Because Lasso regularization is problematic with high degrees of collinearity, we also include the Elastic Net estimator. Finally, we examine the prevalence of Type I (wrongly including a noise predictor) and Type II (wrongly excluding a true predictor) error rates across a range of sample sizes and effect sizes.

To test each form of estimation, we used two different packages in the R statistical environment (R Core Team, 2018). For MLE, we used the lavaan package (version 0.523.1097; Rosseel, 2012). For RegSEM, we used the regsem package (version 1.0.6; Jacobucci, Grimm, Brandmaier, \& Serang, 2017). Both Lasso and Elastic Net regularization are implemented in regsem, along with a host of additional penalties (Jacobucci, 2017). We vary the penalty term lambda (see equation 4 above) across 30 values, ranging from 0 to 0.29 in equal increments. In initial pre-runs, higher penalty values were used but always resulted in worse fit at the higher ranges. To choose a final model among the 30 models run, we used the Bayesian information criteria (Schwarz, 1978). Across all of the simulation conditions, each cell was replicated 200 times. Our simulation code and other material can be found at https://osf.io/z2dtq/.

\section{Results}


Instead of giving a detailed analysis of each figure, we instead give a high level overview of simulation results. We compare the performance of RegSEM Lasso to MLE across three performance metrics: Root Mean Square Error (RMSE; averaged across each set of parameters), relative bias (RB; averaged across each set after taking the absolute value of each parameter) and and error type (type I and type II respectively). For each performance metric we vary sample sizes (left panels) and collinearity (right panels). We do not present the results for RegSEM Elastic Net estimation, as the results were almost identical to those from RegSEM Lasso. 
RMSE v.s. sample size

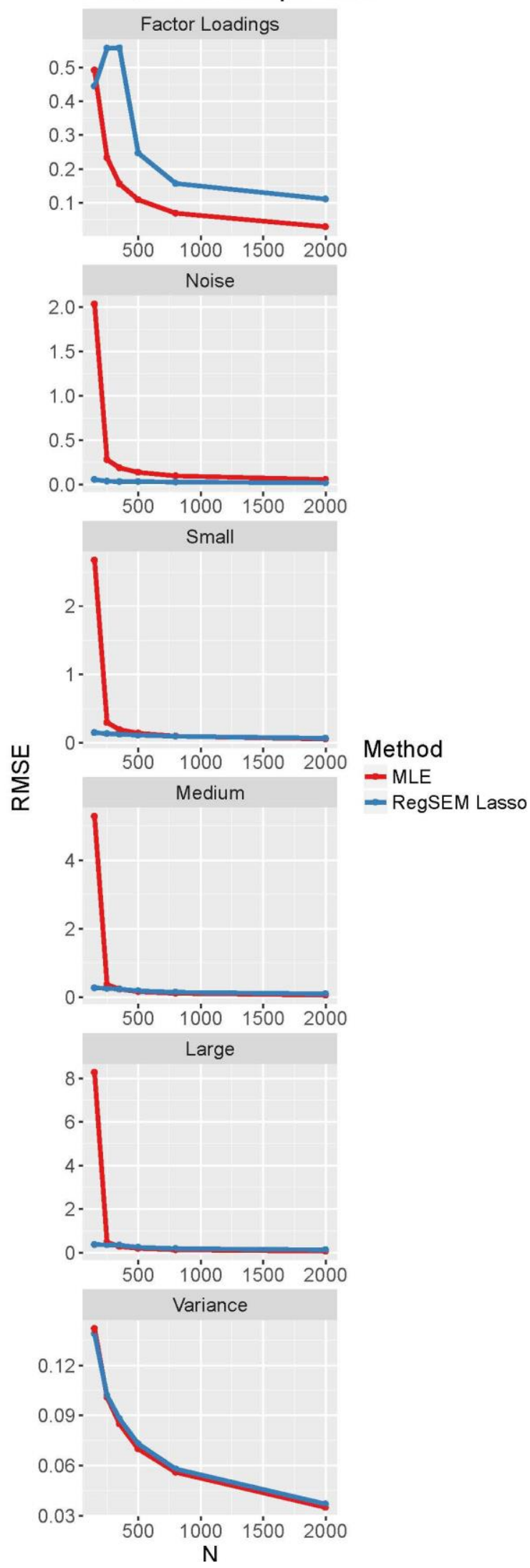

RMSE v.s. collinearity
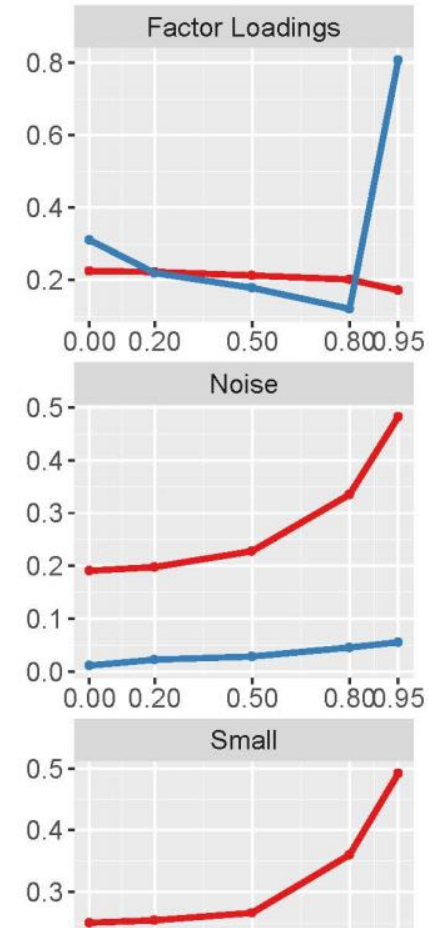

$0.2-$

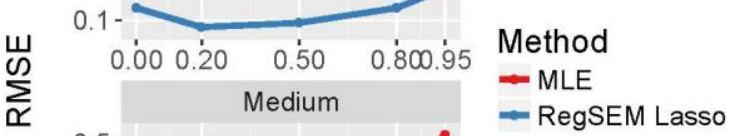

Figure 4: Root mean square error across a range of sample size (left panels) and predictor collinearities (right panels) for MLE (red) and RegSEM lasso (blue). ). The individual panels refer to the factor loadings, the uninformative predictors (noise), to the informative predictors of different effect sizes (small, medium, strong), and the residual error variances (variances). 
Parameter Estimates. First we examine the precision of parameter recovery quantified as RMSE and relative bias (RB). At higher sample sizes, MLE performed well in comparison to Lasso with regard to RMSE, and even more so for RB. This performance distinction RegSEM Lasso between both metrics is as expected, because as we discussed earlier, the Lasso imparts bias to reduce variance. RMSE measures both bias and variance, while RB only measures bias, thus the increase in bias is somewhat offset by a decrease in variance. At smaller sample sizes, the Lasso performed better than MLE, particularly at a sample size of 150 . With only 150 observations, MLE was highly unstable in its estimation of parameters, meaning parameter estimates were drastically larger than their simulated values. In using RMSE, there was remarkably similar performance across both MLE and Lasso, with the exception of sample sizes of 150 and 250. Lasso was favoured with respect to RB for the sample size of 150, but MLE was favoured with larger samples. Using the RMSE, the Lasso produced better results in most conditions, whereas the results with RB were more mixed. Somewhat surprisingly, when the amount of correlation among all predictors was extremely high (0.95), the Lasso produced a large amount of RB in the factor loadings. This secondary effects was much less the present using MLE. Together, our simulations show that regularized SEM outperforms traditional MLE in terms of parameter estimation in cases where sample sizes are small and the number of predictors is large. 

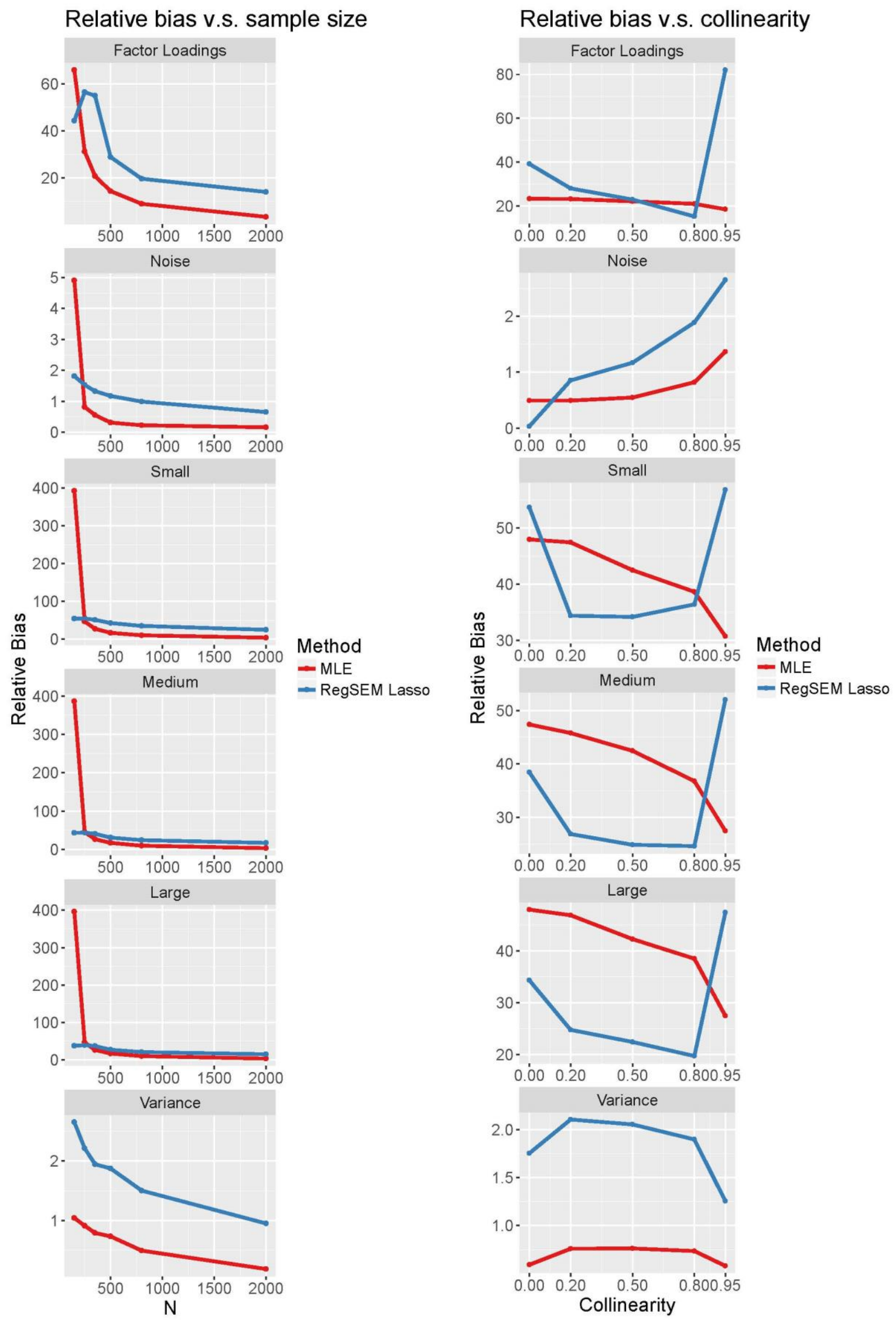

Figure 5: Relative bias across a range of sample size (left panels) and predictor collinearities (right panels) for MLE (red) and RegSEM lasso (blue). 
Type I and Type II Errors. An alpha criterion of 0.05 was used to determine parameter significance in the MLE models (see Figure 6). First looking at the propensity of a Type I error with the noise parameters (if a noise variable had a p-value $<0.05$ ), sample size had a larger effect than did collinearity for MLE. For a sample size of 150, this means a $17 \%$ chance to incorrectly identify a noise variable as a significant parameter. For collinearity, although the Type I errors rates were higher than 0.05 , this can mostly be attributed to the influence of the sample size conditions. More alarming is the low power, or high Type II error rates (p-value > 0.05) for the small and medium parameters in MLE. As collinearity increases, so does the Type II error rates for these parameters, while the inverse relationship holds for sample size. Even for the parameters simulated at a value of 0.8 , larger than expected numbers of Type II errors were committed at small sample sizes and a large amount of collinearity. For the Lasso, almost opposite results occurred. Overall, the Lasso committed far more Type I errors with the noise variables (estimating noise variables as non-zero), but also had much lower Type II errors (i.e., it rarely omitted a truly predictive variable) across the small, medium and large variables in each condition.

\section{Summary}

Across our simulations, MLE performed better at larger sample sizes, the Lasso better at smaller numbers of observations. Across both metrics, MLE had less relative bias (as expected), while in some cases the Lasso improved upon MLE with respect to the RMSE. These results are in line with previous work such as Serang, Jacobucci, Brimhall, and Grimm (2017), who found a similar tradeoff between regularization and other forms of estimation in the context of mediation models. Parameter estimate accuracy had a less stark contrast in the performance between methods. The optimal method for a given research context depends on the relative importance of 
decreasing parameter bias or parameter variance. Although MLE may produce more accurate results within your sample, this model may not generalize as well as a model produced using regularization. This contrast goes beyond small selection of models discussed in this paper. 

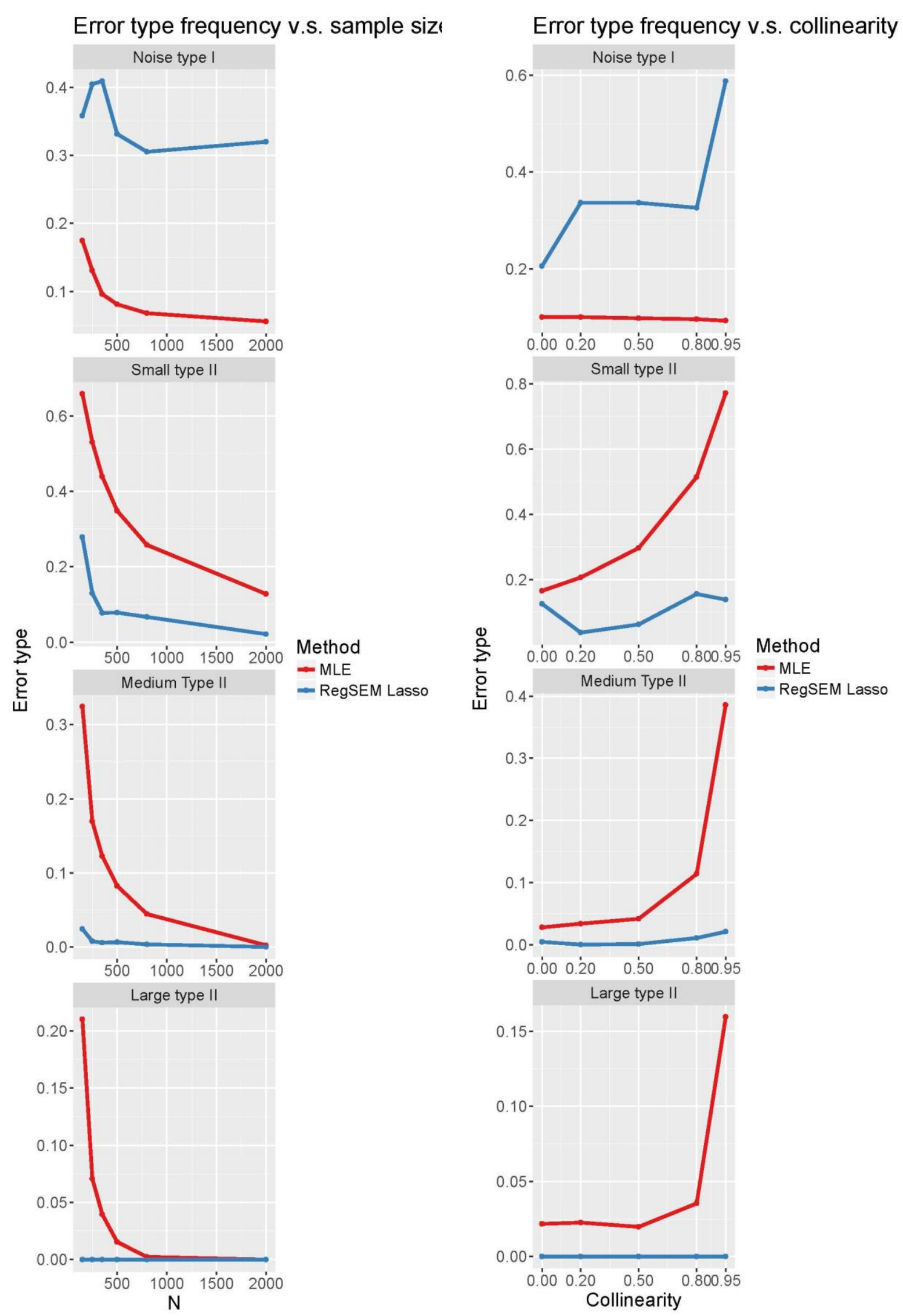

Figure 6: Type I and Type II errors for predictors of small (top) medium (middle) and large (bottom) size, tested across a range of sample size (left panels) and predictor collinearities (right panels) for MLE (red) and RegSEM lasso (blue). 


\section{Empirical application: white matter determinants visual short term memory}

In cognitive neuroscience, where many features of brain structure and function may have complementary effects, the challenge is how to best reconcile the dimensionality constraints for covariance based methodologies such as SEM, with the richness of the imaging metrics (which may include hundreds of measures per individual). Here, we describe an illustrative example using regularized SEM on a large, population-derived cohort of healthy aging individuals (CamCAN, Shafto et al., 2014), modelling visual short term memory as a function of white matter microstructure.

\section{Sample}

For this empirical illustration we use data from the Cambridge Study of Cognition, Aging and Neuroscience (Cam-CAN, www.cam-can.org). The sample consists of 627 participants, 320 female, between 18 and $88(\mathrm{M}=54.18, \mathrm{SD}=18.42)$ who participated in a large battery of cognitive tests, demographic and lifestyle measurements and MRI scans (for more detail on the cohort and sampling methodology see Taylor et al., 2017). Here we focus on a specific cognitive task (the visual short term memory task) and a common index of white matter microstructure (Fractional Anisotropy, FA) for participants with complete data. Subsets of this data (but not this cognitive task) have previously been reported (e.g. Henson et al., 2016; Kievit et al., 2014, 2016). 


\section{Visual Short Term Memory}

This particular visual short term memory

task was developed to quantify capacity and

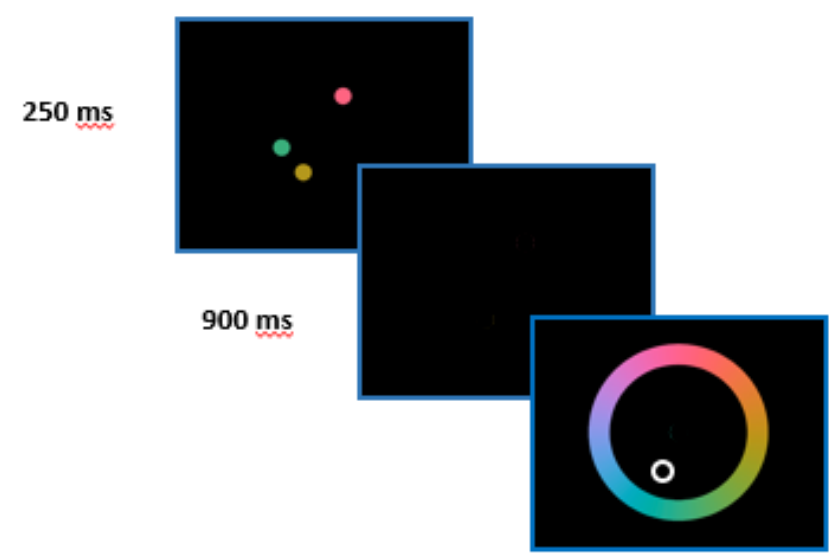

between one and four coloured circles,

followed by a brief blank screen (900

Figure 7: Visual short term memory task. Participants view between 1 and 4 targets for 250 milliseconds, followed by a 900 ms blank screen. Finally they receive a cue for one of the previous targets, and are milliseconds) and a cue in the same spatial asked to respond, using a colour wheel, which hue most closely matched that of the target.

location as one of the (up to) four circles

(see Figure 7). Participants are asked to use a colour wheel to pick the colour of the cued circle, as well as rate their confidence in their judgment. Participants perform a total of 224 trials across two blocks, with position, set size and cues counterbalanced across blocks. We here focus only on set size (defined as the visual capacity of an individual estimated for each set size) for set sizes 2-4 (to avoid the ceiling effects associated with the simplest version). Each participant had three scores capturing their mean performance across the three set sizes, with each score ranging between 0 and the maximum number of circles per set size (i.e. 2-4).

\section{White matter}

For the neural indicators we use a common metric of white matter organization called Fractional Anisotropy. This metric quantifies the dispersion of water molecules and the extent to which this dispersion constrained by the organization of white matter structures. FA is a complex and indirect measure with various limitations, and the relationship between FA and white-matter health is not yet fully understood (Jones, Knösche, \& Turner, 2013; Bender, Prindle, 
Brandmaier, \& Raz, 2016). Nonetheless, FA is widely used as it has been shown to be associated with individual differences in a range of cognitive domains, especially in old age (Madden et al., 2009). We here focus on mean FA for each tract using the ICBM-DTI-81 atlas (Mori et al., 2008) which parcellates the human white matter skeleton in 48 tracts. Although we have used a previously focused on white matter atlases of lower dimensionality (e.g. (Kievit et al., 2016 and Mooij, Henson, Waldorp, Cam-CAN, \& Kievit, 2018), we here intentionally use a more high-dimensional white matter tract atlas to illustrate the benefit of regularization. For more details regarding the pipeline, see (Kievit et al., 2016).

\section{MIMIC-model}

To examine the neural determinants of visual short term memory we fit a Multiple Indicator, Multiple Causes model (Joreskog \& Goldberger, 1975). This model captures the hypothesis that a latent variable measured by multiple indicators is in turn affected by multiple causes (cf. (Kievit et al., 2012) for a comparison of the MIMIC model to competing representations First, we specify a measurement model such that a latent variable is measured by the memory capacity across three subtests varying in set size (2, 3 and 4, see above for more details). Next, we simultaneously regress this latent variable on all 48 white matter tracts. This model tests the joint prediction of the latent variable by all 48 tracts which allows one to quantify if one or more white matter tracts help predict individual differences in visual short term memory.

\section{Model estimation and results}


We estimate the regularized model across a range of lambda values, using the Bayesian Information Criterion (BIC; also Schwarz Criterion) to compare model fit across each iteration. The BIC balances the extent to which the increased parsimony of regularizing parameters to 0 simplifies the model with the concurrent decrease in explanatory power of the reduced model. As we have a strong a priori hypothesis about the measurement model we only regularize the structural parameters (i.e. the joint prediction of the latent variable by 48 tracts), not the factor loadings or residual variances. As can be seen in Figure 8, the best solution by BIC is obtained with a lambda value of 0.18 , which yields an acceptable RMSEA of 0.0321 . Figure 9 shows the beta estimates and model BIC across a range of Lambdas, as well as the six tracts that are nonzero in the final model.

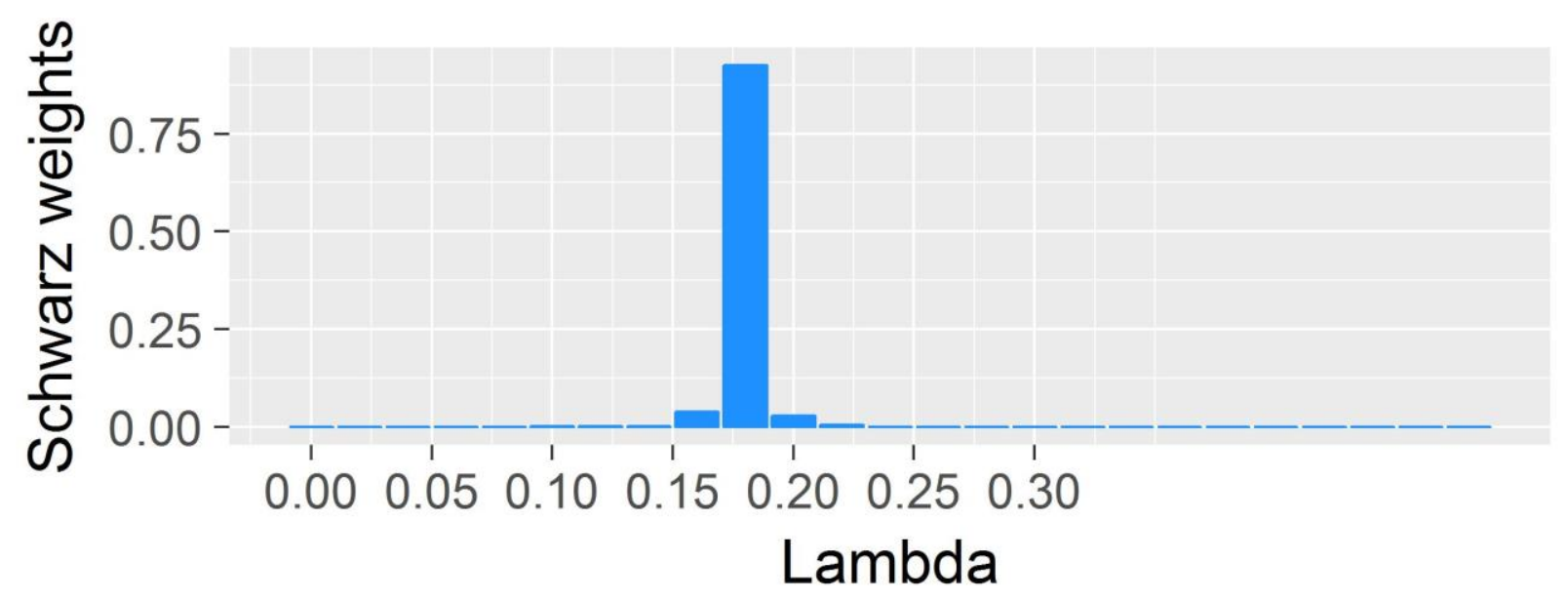

Figure 8: Schwarz Weights (cf. Wagenmakers \& Farrell, 2004) across a range of lambda values, suggesting a lambda of .18 is optimal.

\section{Results}

As can be seen in Figure 9, six tracts remain non-zero in the regularized MIMIC model.

Strikingly, three of these are subdivisions of the fornix (the column and body, as well as the cres), all showing positive effects (i.e. greater white matter microstructure is associated with better visual short term memory performance). The fornix, a tract connecting the hippocampus to 
other brain regions, has long been associated with various aspects of memory, usually autiobiographic (e.g. Hodgetts et ell., 2016) or, in the same Cam-CAN cohort, subdomains such as recollection, familiarity and priming (Henson et al., 2016). Notably, there are even some phase I trials suggesting deep brain stimulation to the fornix may alleviate memory complaints in early Alzheimer's sufferers (Laxton et al., 2010). The posterior thalamic radiations (see Figure 9 top right) have been posited as crucial in focusing and allocation attention in demanding tasks (Menegaux et al., 2017), and evidence in infants suggests distinctive association between greater white matter organization in the posterior thalamic radiations and better performance on the visual short term memory task (Menegaux et al., 2017). Finally, we observe a positive association between the superior fronto-occipital fasciculus, in line with findings in children of greater spatial working memory (Vestergaard et al., 2011). Anatomically this finding makes sense for a demanding visual short term working memory task, as the SFOF tract is a (small) part of a long tract that connects early visual areas in the occipital lobe to control networks in the frontal lobe.
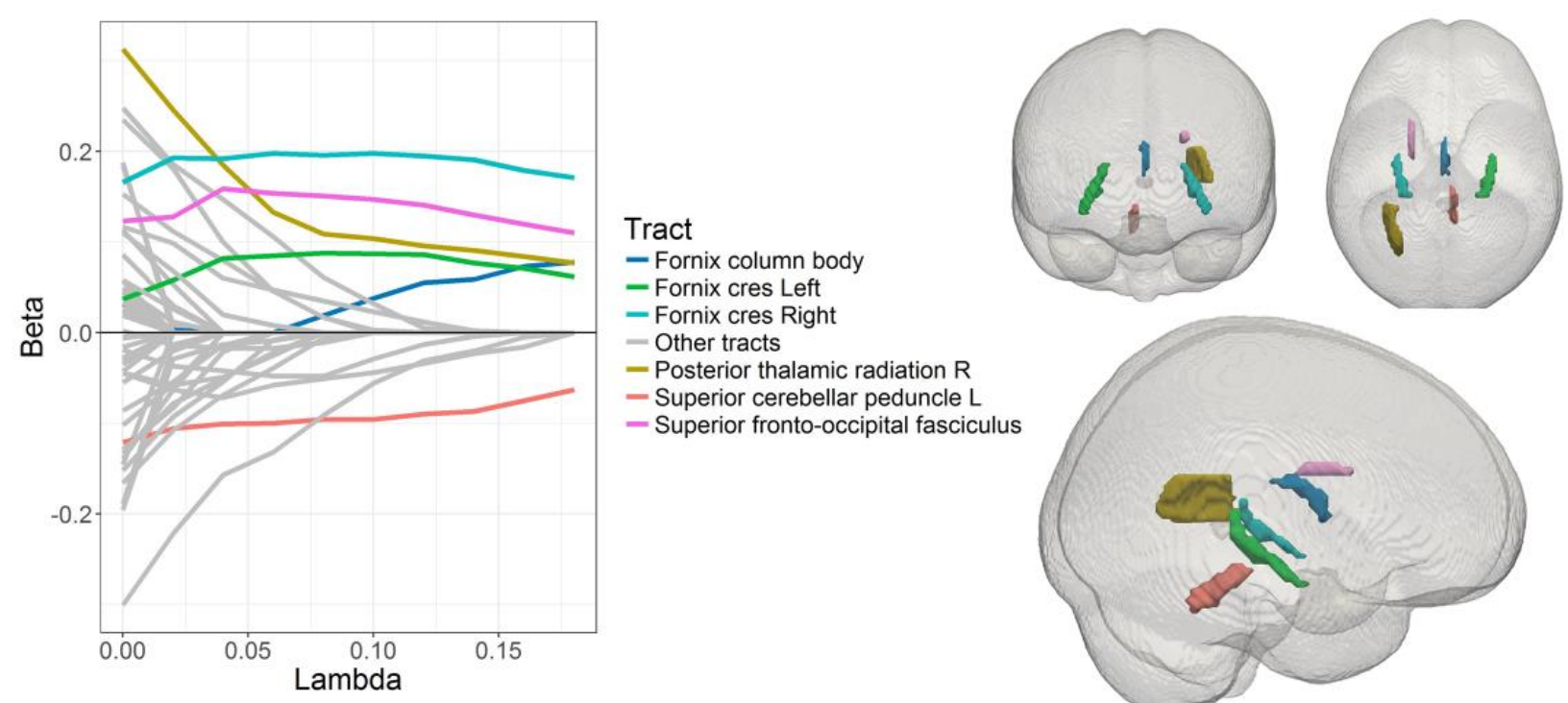
- Fornix cres Right - Other tracts - Posterior thalamic radiation $\mathrm{R}$ - Superior cerebellar peduncle L - Superior fronto-occipital fasciculus

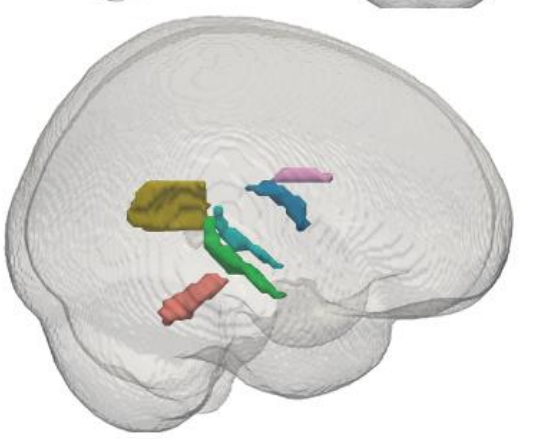

Figure 9: In the final model six non-zero tracts for this penalty are shown as individual colours (top left and top right panels) whereas the tracts regularized to 0 are shown in grey. 
Although the above five tracts align well with previous literature, we also observe a single (surprising) negative effect - Greater white matter integrity of the Superior cerebellar peduncle was associated with poorer VSTM performance. However, closer inspection of this tract suggests this is pattern is likely an artefact of image registration as (unlike other tracts) the integrity of this tract (bilaterally) increases with age. A likely explanation is that the relatively deep location of this tract within the brain makes it vulnerable to registration challenges such as partial volume effects (Alexander, Hasan, Lazar, Tsuruda, \& Parker, 2001) For these reasons we suggest this 'negative' pattern is more likely to represent an imaging artefact than a true association.

Other tracts commonly observed to be associated with working memory performance in aging were not found to be predictors here, most notably white matter organization in the prefrontal lobe (e.g. Kennedy \& Raz, 2009). It should be noted that the regularized model solution does not imply that all other tracts are uncorrelated to VSTM: In cases of collinear predictors a regularized solution is more likely to yield a predictor most 'representative' of a broader set of correlated predictors (i.e. a single tract captures most or all of the predictive power across a network of tracts). To summarize, a regularized SEM-MIMIC is able to model the relation between cognitive performance and imaging metrics with a more high dimensional set of predictors into a relatively parsimonious representation of key tracts previously implicated in visual short term memory performance, providing proof of principle of the viability of this methodology in cognitive neuroscience in general and aging and developmental cohorts in particular.

\section{Discussion}

Here we propose regularized SEM as a powerful and underutilized method for researchers who want to examine a (relatively) large number of predictors, or have a relatively 
modest sample size in SEMs of moderate complexity. We described regularization as applied to both regression and structural equation modeling, and evaluated its use in high dimensional MIMIC models. We show how Lasso penalties incurred less error and bias in conditions with small sample sizes, and demonstrate higher power in detecting regression paths of varying magnitude. Across these results we identified how sample size and the correlation among regressors influences the accuracy and inference of parameter estimates in an extremely complex model. Finally, this was applied to modeling visual short term memory as a function of white matter microstructure in a large existing dataset. Starting with a complex model of 48 distinct white matter tracts, the regularized model yielded six distinct tracts with non-zero parameter estimates as determinants of visual short term memory.

Both studies showed that regularized SEM may be a viable option for researchers looking to identify a relatively low-dimensional set of predictors in fields with broad sets of candidate variables, such as cognitive neuroscience and behavior genetics. Notably, this technique goes beyond traditional mass univariate methods of multiple comparison corrections in neuroimaging such as FDR or Gaussian random field theory (for an accessible introduction, see Brett et al., 2003), which are generally still implemented to correct (mass) univariate tests, rather than joint simultaneous prediction across voxels/regions of interest. It may be possible to combine the above approach with joint methods such as principal component regression to estimate the joint prediction of multiple components across many voxels even in cases with modest sample sizes (e.g., Wager et al., 2011).

\section{Limitations and challenges}

Although we have illustrated several benefits of regularization in regression and SEM for small sample sizes, we did not include any conditions with $\mathrm{N}<100$. This was mostly due to the 
complexity of our model, as we were unable to achieve stable estimates at a sample size of 120 or below. In regularized regression it is possible to test models with $\mathrm{P}>\mathrm{N}$ (where $\mathrm{P}$ is the number of predictors and $\mathrm{N}$ is the sample size), however, to our knowledge, this has not been done using traditional SEM estimation methods such as MLE. A possible solution is the use of Bayesian SEM, where strongly informative priors or hierarchal models with sparsity inducing priors can achieve stable estimation even in such extreme cases (see Jacobucci \& Grimm, in press). Given the use of Bayesian estimation in cases of small numbers of observations (McNeish, 2016), we expect to see more research in this realm in the future. Other avenues for future work include the investigation of bias in the use of regularization in factor score regression approaches (DeVlieger \& Rosseel, 2017), which may help overcome the current N>P boundary.

Without p-values or confidence intervals accompanying each parameter estimate, researchers may feel less certain regarding inference when using regularization. Although Lockhart, Taylor, Tibshirani, and Tibshirani (2014) have derived sampling distributions to calculate p-values that take into account the adaptive nature of the Lasso regression model, this has not been done with RegSEM Lasso. Because of this, inference can be more challenging in regularized SEM models, particularly given the inherent bias in estimation. One method proposed for overcoming this is the relaxed Lasso (Meinshausen, 2007), which has been shown to produce unbiased parameter estimates with the Lasso applied to mediation models (Serang, Jacobucci, Brimhall, \& Grimm, 2017). Despite this, it may be difficult to change one's mindset in characterizing non-zero paths as important. For this, we recommend a conceptualization that relates to an alternative sample. Although we may incur bias through the use of regularization, our more important aim is that of generalization, or reducing variance. This particularly holds in 
exploratory studies, where we are less concerned with within sample inference, and care more about using our model to inform future research.

In our simulation we found a tradeoff between MLE and RegSEM Lasso with respect to Type I and Type II errors - The RegSEM Lasso keeps more variables in the model (more Type I errors and less Type II), where MLE is more restrictive with respect to which variables are thought to be significant (Less Type I and more Type II). Our perspective is that in exploratory studies, more weight should be given to the inclusion of potentially important variables, and less weight to possibly including variables that do not have either predictive or inferential value. In an ideal setting, researchers would apply regularized SEM to data from a pilot or initial study in the hopes of being maximally efficient in what variables are included in a future, possibly larger study. Our simulation study supports that idea that applying MLE when the sample is small and the number of variables is large will result in the exclusion of potentially relevant variables. Note however, that our conclusions rely not only on the choice of regularization but also on our specific heuristic for choosing lambda. If researchers can afford to be more inclusive (i.e. can tolerate more Type I errors) or more exclusive (can tolerate more Type II errors) in variable selection, choosing different lambdas may align better with their goals.

\section{Other approaches}

Regularized SEM is only one of the new methods developed for structural equation modeling in larger datasets. Particularly in the area of variable selection, Structural Equation Model Trees (SEM trees; Brandmaier, von Oertzen, McArdle, \& Lindenberger, 2013) and Forests (Brandmaier, Prindle, McArdle, \& Lindenberger, 2016) are one alternative method. SEM trees directly use the observed covariates to partition observations and in the process, only a subset of covariates are used to create a tree model, allowing researchers to uncover non-linearites and 
interactions in Structural Equation Models. Additional methods include the use of heuristic search algorithms (e.g. Marcoulides \& Ing, 2012), various methods for identifying group differences (Frick, Strobl, \& Zeileis, 2015; Kim \& von Oertzen, 2017; Tutz \& Schauberger, 2015), and the use of graphical models for identifying latent variables (e.g Epskamp, Rhemtulla, \& Borsboom, 2016). With increasing amounts of data sharing, facilitated by various new tools for data storage and sharing such as the Open Science Framework (https://osf.io/) and https://openfmri.org/ we can envision the utility of testing models much larger than our template simulation model. One of the biggest challenges is current software implementations. In this regard we expect Bayesian estimation to be particularly fruitful, especially creation of new sampling methods such as in the Stan software package (Carpenter et al., 2016), with easier to use interfaces for specifying models (see Merkle \& Rosseel, 2015), is sure to facilitate wider use among psychological researchers. As discussed earlier, regularization can be accomplished through both frequentist and Bayesian estimation (see Jacobucci \& Grimm, in press), with varying strengths and weakness to each approach.

\section{Summary}

We encourage researchers to think of regularization as an approach to allow the incorporation of confirmatory and exploratory modeling. Researchers have more flexibility to make both their uncertainty and knowledge concrete. This is particularly suitable if researchers hope to use a principled approach to go beyond what the limitations to their theory to identify potentially fruitful avenues for future study. In both our simulation and empirical example, we did an exploratory search for important predictors in relation to a confirmatory latent variable model. This is only one example in the fusion of these types of modeling and we look forward to see 
new areas for application. It is our hope that our exposition shed light on a new family of statistical methods that have a high amount of utility for use in psychological datasets. 


\section{References}

Alexander, A. L., Hasan, K. M., Lazar, M., Tsuruda, J. S., \& Parker, D. L. (2001). Analysis of partial volume effects in diffusion-tensor MRI. Magnetic Resonance in Medicine, 45(5), 770-780. http://doi.org/10.1002/mrm.1105

Bakker, M., Van Dijk, A., \& Wicherts, J. M. (2012). The rules of the game called psychological science. Perspectives on Psychological Science, 7, 543-554.

Bender, A. R., Prindle, J. J., Brandmaier, A. M., \& Raz, N. (2016). White matter and memory in healthy adults: Coupled changes over two years. NeuroImage, 131, 193-204. doi:10.1016/j.neuroimage.2015.10.085

Benjamini, Y., \& Hochberg, Y. (1995). Controlling the false discovery rate: a practical and powerful approach to multiple testing. Journal of the royal statistical society. Series B (Methodological), 289-300.

Brandmaier, A. M., von Oertzen, T., McArdle, J. J., \& Lindenberger, U. (2013). Structural equation model trees. Psychological methods, 18(1), 71.

Brandmaier, A. M., Prindle, J. J., McArdle, J. J., \& Lindenberger, U. (2016). Theory-guided exploration with structural equation model forests. Psychological Methods, 21(4), 566.

Brandmaier, Wenger, Raz, \& Lindenberger (2018). Assessing reliability in neuroimaging research through intra-class effect decomposition (ICED). Submitted manuscript.

Brett, M., Penny, W., \& Kiebel, S. (2003). Introduction to random field theory. Human brain function, 2 .

Carpenter, B., Gelman, A., Hoffman, M., Lee, D., Goodrich, B., Betancourt, M., ... Riddell, A. (2016). Stan: A probabilistic programming language. Journal of Statistical Software, 20, $1-37$. 
Epskamp, S., Rhemtulla, M., \& Borsboom, D. (2016). Generalized network psychometrics: Combining network and latent variable models. Psychometrika, 1-24.

Frick, H., Strobl, C., \& Zeileis, A. (2015). Rasch mixture models for DIF detection: A comparison of old and new score specifications. Educational and Psychological Measurement, 75(2), 208-234.

Grice, J. W. (2001). Computing and evaluating factor scores. Psychological Methods, 6(4), 430.

Grömping, U. (2009). Variable importance assessment in regression: linear regression versus random forest. The American Statistician, 63(4), 308-319.

Harrell Jr, F. E. (2015). Regression modeling strategies: with applications to linear models, logistic and ordinal regression, and survival analysis. (2 ${ }^{\text {nd }}$ Edition). Springer.

Hastie, T., Tibshirani, R., \& Wainwright, M. (2015). Statistical learning with sparsity: the lasso and generalizations. CRC press.

Helwig, N. E. (2017). Adding bias to reduce variance in psychological results: A tutorial on penalized regression. The Quantitative Methods for Psychology, 13(1), 1-19.

Henson, R. N., Campbell, K. L., Davis, S. W., Taylor, J. R., Emery, T.,

Erzinclioglu, S., ... Kievit, R. A. (2016). Multiple determinants of lifespan memory differences. Scientific Reports, 6(1), 32527. http://doi.org/10.1038/srep32527

Hirose, K., \& Yamamoto, M. (2015). Sparse estimation via nonconcave penalized likelihood in factor analysis model. Statistics and Computing, 25(5), 863-875.

Hodgetts, C. J., Postans, M., Warne, N., Varnava, A., Lawrence, A. D., \& Graham, K. S. (2017). Distinct contributions of the fornix and inferior longitudinal fasciculus to episodic and semantic autobiographical memory. cortex, 94, 1-14. 
Huang, P.-H., Chen, H., \& Weng, L.-J. (2017). A penalized likelihood method for structural equation modeling. Psychometrika, 1-26.

Jacobucci, R. (2017). regsem: Regularized Structural Equation Modeling. arXiv preprint arXiv:1703.08489.

Jacobucci, R., \& Grimm, K. J. (in press). Regularized estimation of multivariate latent change score models. In E. Ferrer, S. Boker, \& K. J. Grimm (Eds.), Advances in Longitudinal Models for Multivariate Psychology: A Festschrift for Jack McArdle. Routledge, London.

Jacobucci, R., \& Grimm, K. J. (in press). Comparison of frequentist and Bayesian regularization in structural equation modeling. Structural Equation Modeling: A Multidisciplinary Journal

Jacobucci, R., Grimm, K. J., \& McArdle, J. J. (2016). Regularized structural equation modeling. Structural Equation Modeling: A Multidisciplinary Journal, 23, 555-566.

Jacobucci, R., Grimm, K. J., Brandmeier, A. M., \& Serang, S. (2017). regsem: Regularized structural equation modeling. $\mathrm{R}$ package version 1.0.6. Retrieved from https://cran.rproject.org/package=regsem

James, G., Witten, D., Hastie, T., \& Tibshirani, R. (2013). An Introduction to Statistical Learning. Springer, New York, New York..

Jones, D. K., Knösche, T. R., \& Turner, R. (2013). White matter integrity, fiber count, and other fallacies: The do's and don'ts of diffusion MRI. NeuroImage.

Jöreskog, K. G., \& Goldberger, A. S. (1975). Estimation of a model with multiple indicators and multiple causes of a single latent variable. Journal of the American Statistical Association, 70, 631-639. 
Kievit, R. A., van Rooijen, H., Wicherts, J. M., Waldorp, L. J., Kan, K.-J., Scholte, H. S., \& Borsboom, D. (2012). Intelligence and the brain: A model-based approach. Cognitive Neuroscience, 3(2), 89-97. http://doi.org/10.1080/17588928.2011.628383

Kievit, R. A., Davis, S. W., Mitchell, D., Taylor, J. R., Duncan, J., Cam-CAN, \& Henson, R. N. (2014). Distinct aspects of frontal lobe structure mediate age-related differences in fluid intelligence and multitasking. Nature Communications, 5(5658), 1-10.

Kievit, R. A., Davis, S. W., Griffiths, J., Correia, M. M., Cam-Can, \& Henson, R. N. (2016). A watershed model of individual differences in fluid intelligence. Neuropsychologia, 91, 186-198. http://doi.org/10.1016/j.neuropsychologia.2016.08.008

Kim, B., \& von Oertzen, T. (2017). Classifiers as a model-free group comparison test. Behavior Research Methods, 1-11.

Kline, R. B. (2015). Principles and practice of structural equation modeling. Guilford.

Laxton, A. W., Tang-Wai, D. F., McAndrews, M. P., Zumsteg, D., Wennberg, R., Keren, R., ... \& Lozano, A. M. (2010). A phase I trial of deep brain stimulation of memory circuits in Alzheimer's disease. Annals of neurology, 68(4), 521-534.

Lockhart, R., Taylor, J., Tibshirani, R. J., \& Tibshirani, R. (2014). A significance test for the lasso. Annals of Statistics, 42(2), 413.

MacCallum, R. C., Roznowski, M., \& Necowitz, L. B. (1992). Model modifications in covariance structure analysis: the problem of capitalization on chance. Psychological Bulletin, 111(3), 490.

Madden, D. J., Spaniol, J., Costello, M. C., Bucur, B., White, L. E., Cabeza, R., ... Huettel, S. A. (2009). Cerebral white matter integrity mediates adult age differences in cognitive performance. Journal of Cognitive Neuroscience, 21(2), 289-302. 
http://doi.org/10.1162/jocn.2009.21047

Marcoulides, G. A., \& Ing, M. (2012). Automated structural equation modeling strategies. In R. Hoyle (Ed.), Handbook of tructural equation modeling (pp. 690-704). New York, NY:.Guilford.

McNeish, D. M. (2015). Using lasso for predictor selection and to assuage overfitting: A method long overlooked in behavioral sciences. Multivariate Behavioral Research, 50(5), 471-484.

McNeish, D. M. (2016). On using Bayesian methods to address small sample problems. Structural Equation Modeling, 23(5), 750-773. doi:10.1080/10705511.2016.1186549

Meehl, P. E. (1990). Why summaries of research on psychological theories are often uninterpretable. Psychological Reports, 66(1), 195-244.

Meinshausen, N. (2007). Relaxed lasso. Computational Statistics \& Data Analysis, 52, 374-393. doi:10.1016/j.csda.2006.12.019

Menegaux, A., Meng, C., Neitzel, J., Bäuml, J. G., Müller, H. J., Bartmann, P., ... Sorg, C. (2017). Impaired visual short-term memory capacity is distinctively associated with structural connectivity of the posterior thalamic radiation and the splenium of the corpus callosum in preterm-born adults. NeuroImage, 150, 68-76.

http://doi.org/10.1016/j.neuroimage.2017.02.017

Merkle, E. C., \& Rosseel, Y. (2015). blavaan: Bayesian structural equation models via parameter expansion. arXiv preprint arXiv:1511.05604.

Mooij, S. M. M. de, Henson, R. N. A., Waldorp, L. J., Cam-CAN, -, \& Kievit, R. A. (2017). Age differentiation within grey matter, white matter and between memory and white matter in an adult lifespan cohort. bioRxiv, 148452. http://doi.org/10.1101/148452 
Mori, S., Oishi, K., Jiang, H., Jiang, L., Li, X., Akhter, K., ... Mazziotta, J. (2008). Stereotaxic white matter atlas based on diffusion tensor imaging in an ICBM template. NeuroImage, 40(2), 570-582. http://doi.org/10.1016/j.neuroimage.2007.12.035

Park, M. Y., \& Hastie, T. (2007). L1-regularization path algorithm for generalized linear models. Journal of the Royal Statistical Society: Series B (Statistical Methodology), 69(4), 659-677.

Pfefferbaum, A., Sullivan, E. V, Hedehus, M., Lim, K. O., Adalsteinsson, E., \& Moseley, M. (2000). Age-related decline in brain white matter anisotropy measured with spatially corrected echo-planar diffusion tensor imaging. Magnetic Resonance in Medicine : Official Journal of the Society of Magnetic Resonance in Medicine / Society of Magnetic Resonance in Medicine, 44(2), 259-68.

R Core Team. (2018). R: A language and environment for statistical computing. Vienna, Austria: R Foundation for Statistical Computing.

Rosseel, Y. (2012). Lavaan: An r package for structural equation modeling. Journal of Statistical Software, 48 (2), 1-36.

Schwarz, G. (1978). Estimating the dimension of a model. The Annals of Statistics, $6(2), 461-464$.

Serang, S., Jacobucci, R., Brimhall, K., \& Grimm, K. J. (2017). Exploratory mediation analysis via regularization. Structural Equation Modeling: A Multidisciplinary Journal, $24,733-744$.

Shafto, M. A., Tyler, L. K., Dixon, M., Taylor, J. R., Rowe, J. B., Cusack, R., ... Henson, R. N. (2014). The Cambridge Centre for Ageing and Neuroscience (Cam-CAN) study protocol: a cross-sectional, lifespan, multidisciplinary examination of healthy cognitive ageing. 
NeuroImage, 14(204), 1-25. http://doi.org/10.1186/s12883-014-0204-1

Skrondal, A., \& Laake, P. (2001). Regression among factor scores. Psychometrika, 66(4), 563-575.

Takahashi, M., Iwamoto, K., Fukatsu, H., Naganawa, S., Iidaka, T., \& Ozaki, N. (2010). White matter microstructure of the cingulum and cerebellar peduncle is related to sustained attention and working memory: A diffusion tensor imaging study. Neuroscience Letters, 477(2), 72-76. http://doi.org/10.1016/j.neulet.2010.04.031

Taylor, J. R., Williams, N., Cusack, R., Auer, T., Shafto, M. A., Dixon, M., ... Henson, R. N. (2017). The Cambridge Centre for Ageing and Neuroscience (Cam-CAN) data repository: Structural and functional MRI, MEG, and cognitive data from a crosssectional adult lifespan sample. NeuroImage, 144(Pt B), 262-269. http://doi.org/10.1016/j.neuroimage.2015.09.018

Thompson, B. (1995). Stepwise regression and stepwise discriminant analysis need not apply here: A guidelines editorial.

Tibshirani, R. (1996). Regression shrinkage and selection via the lasso. Journal of the Royal Statistical Society: Series B, 58, 267-288.

Tutz, G., \& Schauberger, G. (2015). A penalty approach to differential item functioning in Rasch models. Psychometrika, 80(1), 21-43.

Vestergaard, M., Madsen, K. S., Baaré, W. F., Skimminge, A., Ejersbo, L. R., Ramsøy, T. Z., ... \& Jernigan, T. L. (2011). White matter microstructure in superior longitudinal fasciculus associated with spatial working memory performance in children. Journal of Cognitive Neuroscience, 23(9), 2135-2146. 
Wager, T. D., Atlas, L. Y., Leotti, L. A., \& Rilling, J. K. (2011). Predicting individual differences in placebo analgesia: contributions of brain activity during anticipation and pain experience. Journal of Neuroscience, 31(2), 439-452.

Yuan, K. H., Yang, M., \& Jiang, G. (2017). Empirically corrected rescaled statistics for SEM with small $\mathrm{n}$ and large p. Multivariate Behavioral Research, 52(6), 673-698.

Zou, H., \& Hastie, T. (2005). Regularization and variable selection via the elastic net. Journal of the Royal Statistical Society: Series B (Statistical Methodology), 67 (2), $301-320$. 


\section{Footnotes}

1. We tested a sample size of 120 as well, but the regsem package failed to converge at a high rate, thus we did not include these results.

2. Note that we omit the mean structure for pedagogical purposes.

3. Note that regression paths can be penalized regardless of what parameters they come from and are to. For example, they can be from manifest variables to predict latent variables, this directionality can be reversed, between latent variables, as well as only include manifest variables. In fact, Lasso regression can be seen as a subset of RegSEM Lasso. 